\title{
Caracterización espacial de la precipitación en el cañón del rio Cauca, tramo comprendido entre los municipios de Caramanta y Sabanalarga, jurisdicción de Corantioquia, departamento de Antioquia, durante el periodo 2004 - 2014
}

\section{Spatial characterization of rainfall in the Cauca river canyon, section included between the municipalities of Caramanta and Sabanalarga, jurisdiction of Corantioquia, department of Antioquia, during the period 2004-2014}

\author{
Carolina Bahamon Urrea \\ Profesional Corantioquia \\ cbahamon@corantioquia.gov.co
}

\author{
Libardo Antonio Londoño \\ Docente Universidad San Buenaventura \\ libaranto@gmail.com
}

\author{
Arbei Osorio Restrepo \\ Estudiante Doctorado Ingeniería \\ Ambiental UDEA \\ arbei.osorio@udea.edu.co
}

(Tipo de Artículo: Investigación Científica y Tecnológica. Recibido: 25/11/2017. Aprobado: 23/01/2018)

\begin{abstract}
Resumen. El crecimiento poblacional, las actividades económicas intensivas y el cambio climático, han generado una gran presión o estrés sobre el recurso hídrico, evidenciando la necesidad de establecer como unidad de intervención la cuenca hidrográfica e implementar en ella modelos que permitan su ordenación. En este trabajo se generó un nuevo mapa de precipitación media mensual, buscando que este sea incorporado en el modelo existente y posteriormente replicar el procedimiento en las demás zonas que hacen parte de dicha jurisdicción. Los mapas de precipitación obtenidos fueron el resultado de una combinación óptima entre los estimados a partir de las bases de datos del IDEAM de precipitación media mensual y los estimados a partir de la implementación de los modelos aplicados para cada mes. Dicha combinación o ponderación se basó en los campos de varianza del error. De igual manera, se encontró que los modelos j-bessel, hole effect y gaussian fueron los que presentaron mayor frecuencia de todos los modelos evaluados.
\end{abstract}

Abstract. Both big pressure and stress over hydric resource have been generated because of population growth, intensive economic activities and climate change. This situation has shown the need to stablish the watershed as an intervention unit and to implement models in this basin in order to enable its organization. A map of average monthly rainfall was generated in this work, aiming to incorporate it in the existing model and subsequently replicate this procedure on the other zones present in the same jurisdiction. Estimates from IDEAM's data bases of average monthly rainfall and from the implementation of models applied for each month were made in order to obtain the rainfall maps whose combinations and ponderations were based on the error variance fields. Furthermore, among all the models tested the ones with the highest frequency were found to be J-bessel, Hole effect and Gaussian.

Palabras clave. Análisis Espacial, Interpolación Espacial, Precipitación

Keywords. Spatial Analysis, Spatial Interpolation, Rainfall

DOI 10.21500/20275846.3319

\section{Introducción}

El recurso hídrico es indispensable para la supervivencia del hombre y el desarrollo económico de una región, es por ello que su gestión o administración se ha convertido en un tema de alto interés a nivel mundial y ha motivado la búsqueda continua de modelos y herramientas que faciliten la toma de decisiones para garantizar su uso racional y sostenible [1][2].

El crecimiento poblacional, las actividades económicas intensivas y el cambio climático, han generado una gran presión o estrés sobre el recurso hídrico, evidenciando la necesidad de establecer como unidad de intervención la cuenca hidrográfica e implementar en ella modelos que permitan su ordenación y vinculen directamente a las comunidades asentadas en el territorio, con el fin de garantizar un desarrollo sostenible que garanticen el progreso económico y social sin poner en riesgo la existencia del recurso [3].

Para garantizar una gestión o administración integral del recurso hídrico, es importante mantener el equilibrio entre la oferta y la demanda, considerando no solo las asignaciones de caudal para las diferentes actividades del hombre sino también los requerimientos necesarios para mantener los ecosistemas acuáticos y la reserva ecológica [4]. Levite et al, estudiaron la cuenca del rio 
Olifants en África del Sur, mediante la implementación del modelo WEAP, desarrollado por el Instituto de Medio Ambiente de Estocolmo (SEI), en la que encontraron que en períodos secos el riesgo de no poder suplir las necesidades ecológicas en las cuencas e incluso de no poder abastecer en su totalidad a los usuarios con asignaciones de agua preestablecidas aumenta considerablemente, debido esto a la disminución del régimen de lluvias provocado principalmente por el calentamiento global y la deforestación en las cuencas, lo cual se encuentra directamente relacionado con la temperatura y los cambios en el ciclo hidrológico.

Información precisa sobre precipitaciones es de suma importancia para numerosas aplicaciones críticas [5], [6]. Sin embargo, la red mundial de estaciones de lluvia se distribuye de manera desigual e incluso es inexistente para una parte sustancial del globo. La distribución de las estaciones suele estar sesgada, lo que limita nuestra comprensión de los regímenes de lluvias, la variabilidad espacial y temporal y la prevención del desarrollo sistemas de vigilancia fiables en tiempo real [7].

La instalación y mantenimiento de pluviómetros con una adecuada densidad para medir con precisión la precipitación puede resultar una tarea costosa y difícil [8]. Lo pluviómetros son la fuente principal de mediciones de la precipitación directa en América del Sur y son necesarios para calibrar y validar las estimaciones de precipitación obtenidos a partir de mediciones de radar o indirecta vía satélite y modelos numéricos. Sin embargo, las observaciones sobre América del Sur son insuficientes y desigualmente distribuida [9].

En Colombia, a la fecha existen diversos mapas de precipitación que ayudan a la toma de decisiones, pero son pocos los estudios que evalúan la oferta y demanda del recurso. Entre los estudios que abordan la oferta y demanda del recurso hídrico, encontramos que a nivel país, el IDEAM para el año 2014 elaboró el Estudio Nacional del Agua, el cual soporta la evaluación de la situación actual y posibles escenarios futuros del agua en Colombia y tiene como referente base los conceptos y las metodologías del Estudio Nacional del Agua 2010, complementados con aquellos que constituyen temáticas nuevas del estudio [10].

Corantioquia de manera similar cuenta con una herramienta de administración del recurso hídrico superficial, que facilita la toma de decisiones para una buena gestión, denominada Modelo para la Administración del Recurso Hídrico Superficial DUBERDICUS. Este tipo de herramientas permiten la disponibilidad hídrica, en la cual se consideran variables como precipitación, temperatura, evaporación, demanda de agua, planes de desarrollo económico y social, preservación del ambiente y necesidad de mantener reservas suficientes del recurso hídrico [11].

Dentro de los modelos la variable más importante es la precipitación, debido a que es de interés en el análisis hidrológico porque interviene de manera directa en los flujos de materia y energía, generando procesos físicos fundamentales para entender los demás componentes del ciclo hidrológico, como la escorrentía, la recarga, la infiltración y la evapotranspiración [12], [13]. En la actualidad, Corantioquia cuenta con un mapa de precipitación media anual al año 2006 generado mediante el modelo de interpolación Kriging Ordinario, utilizando datos de precipitación de 471 estaciones existentes en el área influencia.

Acorde con el IDEAM, el procedimiento general de espacialización de las variables hidrometeorológicas, se inicia ubicando y seleccionando las estaciones a utilizar en el análisis, para posteriormente procesar la información y elaborar los mapas de cada una de las variables, a la resolución temporal requerida, según corresponda; esta información debe ser vectorizada para su tratamiento en el Sistema de Información Geográfica (SIG) [14].

Para la obtención de los mapas de distribución de la precipitación a cualquier escala temporal frecuentemente se utilizan técnicas geoestadísticas de interpolación como Kriging, este método adquiere gran importancia pues, aunque originalmente fue limitado al campo minero, su uso se ha expandido durante los últimos años para otras ciencias. Especialmente, el método ha encontrado importantes desarrollos en la hidrología. Kriging esencialmente consiste en estimar un valor desconocido de una variable estudiada como una combinación lineal de $\mathrm{n}$ registros de campo de la misma [15].

Por lo anterior, en este trabajo se desarrolló una caracterización de la variable precipitación de los últimos 10 años de las estaciones meteorológicas pertenecientes al IDEAM (Instituto de Hidrología, Meteorología y Estudios Ambientales), con un periodo comprendido entre enero de 2004 a junio de 2014, para lo cual se seleccionó el mejor modelo teórico que representa el cambio de la varianza con la distancia, para luego aplicar el algoritmo de kriging ordinario, mediante la herramienta ARCGIS.

Este estudio se realiza con el fin de generar un nuevo mapa de precipitación media mensual, buscando que este sea incorporado en el modelo existente y posteriormente replicar el procedimiento en las demás zonas que hacen parte de dicha jurisdicción.

\section{2. Área de estudio y características}

El presente estudio se realizó en la franja del Río Cauca específicamente en jurisdicciones de los municipios de Caramanta y Sabanalarga en comunidades que se autodenominan "cañoneras" y que designan al mismo Rio como "El Patrón Mono" por los sedimentos que deposita y por el oro que les brinda [16]. La Franja del cañón está dominado principalmente por un bosque seco tropical el cual se extiende desde Caramanta hasta Sabanalarga como se observa en la Figura 1. 


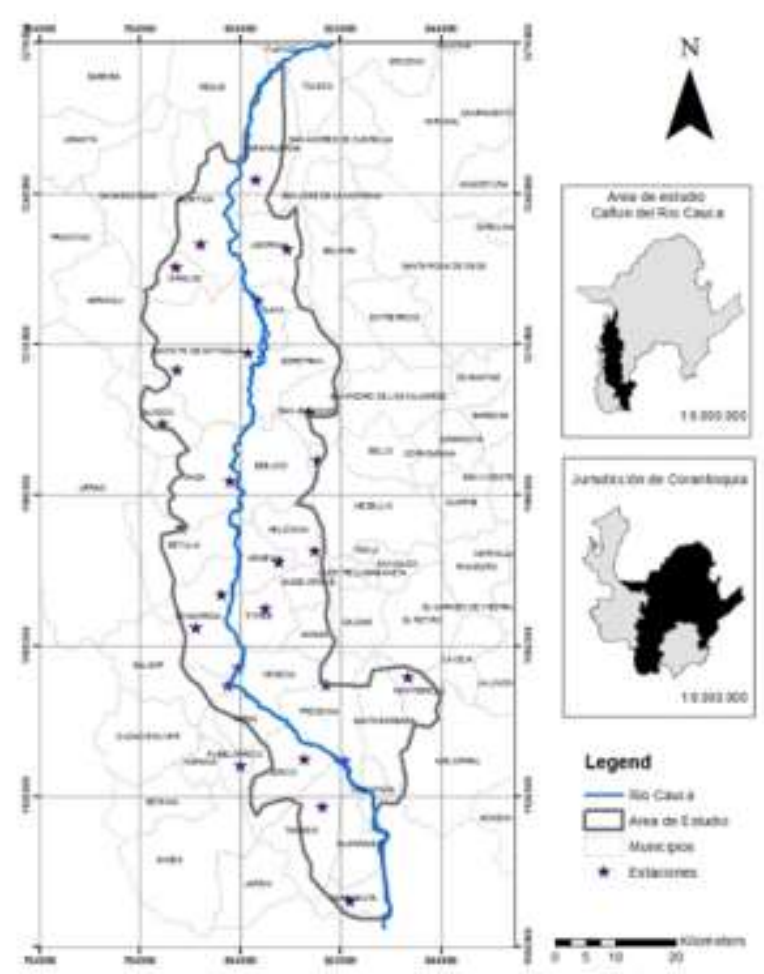

Figura 1. Ubicación de la zona específica del área de estudio Cañón del Rio Cauca

Este cañón sirve de límite natural entre una parte del Noroccidente de Antioquia y las cordilleras centrales y Occidental del país, perteneciente en su gran mayoría a la formación vegetal (Zona de vida) de Bosque Seco Tropical (bs-T), con temperatura media anual superior a los $25^{\circ} \mathrm{C}$ y un promedio anual de precipitación entre los 1000 y 2000 mm, seguido de pequeñas zonas de vida como son el Bosque Húmedo Premontano (bh-PM) precipitación media anual entre 1000 y $2000 \mathrm{~mm}$ y temperatura media anual de 18 a $24^{\circ} \mathrm{C}$, . Bosque muy Húmedo Premontano (bmh-PM) tiene una temperatura promedio anual entre los 18 y $24^{\circ} \mathrm{C}$ y una precipitación media anual entre los 2000 y $4000 \mathrm{~mm}$ y por ultimo pequeñas áreas de Bosque muy Húmedo Montano Bajo (bh-MB) con precipitación anual que varía 1000 y 2000 $\mathrm{mm}$ y una temperatura media anual entre los 12 y $18^{\circ} \mathrm{C}$. Toda la zona oscila entre los 1000 y 3000 msnm [17], [18].

\section{Metodología}

La metodología está implementada en las herramientas Spatial Analyst $\AA^{\circledR}$ y Geostatistical Analyst ${ }^{\circledR}$ del aplicativo ArcMap ${ }^{\circledR}$ de ESRI, se basa en gran parte del trabajo realizado por Londoño et al [19] y Quiroz et al [20]. Para poder aplicar las técnicas geoestadísticas se utiliza la información del valor de precipitación en las estaciones hidrometeorológicas ubicadas en el área de estudio (26 estaciones en total, Fig. 1), utilizando datos promedios mensuales de los últimos 10 años con corte a diciembre de 2014, suministrado por el Instituto de Hidrología, Meteorología y Estudios Ambientales IDEAM. Con este conjunto se datos se hace un análisis exploratorio para validar el cumplimiento del principio de estacionariedad en cada mes. El proceso se muestra en la parte derecha de la fig. 2 [20]. Para los conjuntos de datos en donde el valor absoluto de coeficiente de sesgo (skewness) es mayor o igual a 0.5 se realizan transformaciones según los siguientes criterios [21]:

- Si $0.5<|\mathrm{CS}|<=1$, entonces se aplica una transformación raíz cuadrada.

- Si $|\mathrm{CS}|>1$, entonces se aplica una transformación logarítmica.

Para los conjuntos de datos en los cuales las transformaciones anteriores no mejoran la estacionariedad, se realiza un análisis y detección de valores atípicos. Éstos se retiran de los datos y se realiza nuevamente el análisis exploratorio. Como se muestra en la parte inferior izquierda de la fig. 2, una vez determinado el conjunto de datos estacionarios en cada mes, se hace el análisis estructural de los datos construyendo primero el semivariograma experimental y luego ajustando un modelo de semivariograma teórico. El siguiente paso es determinar cuál es el modelo teórico que mejor representa el cambio de la varianza con la distancia. Se prueban nueve modelos (esférico, gaussiano, tetraesférico, pentaesférico, exponencial, racional cuadrático, hole effect, K-bessel y J-bessel) y luego se aplica el algoritmo de kriging ordinario. Mediante pruebas de validación cruzada usando la técnica "leave one out" [22] y los siguientes criterios [23], se selecciona el mejor modelo teórico:

- Partial sill del modelo similar a la varianza $(\sigma 2)$ de los datos.

- El error estándar promedio entre el dato observado y el dato simulado (ASE average standard error) debe ser pequeño.

- La raíz del error cuadrático promedio (RMS-root mean square-error) debe ser pequeño.

- La raíz del error cuadrático promedio estandarizado debe estar cerca de uno (RMSS-root mean squareerror standarized).

Para los modelos obtenidos se calcula su desempeño y éste se corrige por los efectos de la densificación de los datos mediante la ecuación (1).

$$
\mathrm{Cc}=100-\mathrm{RMS}(1) \text {. }
$$

Seguidamente se hace un análisis de frecuencias con los mejores modelos obtenidos en los 10 años para determinar el modelo mensual más frecuente (modal). 


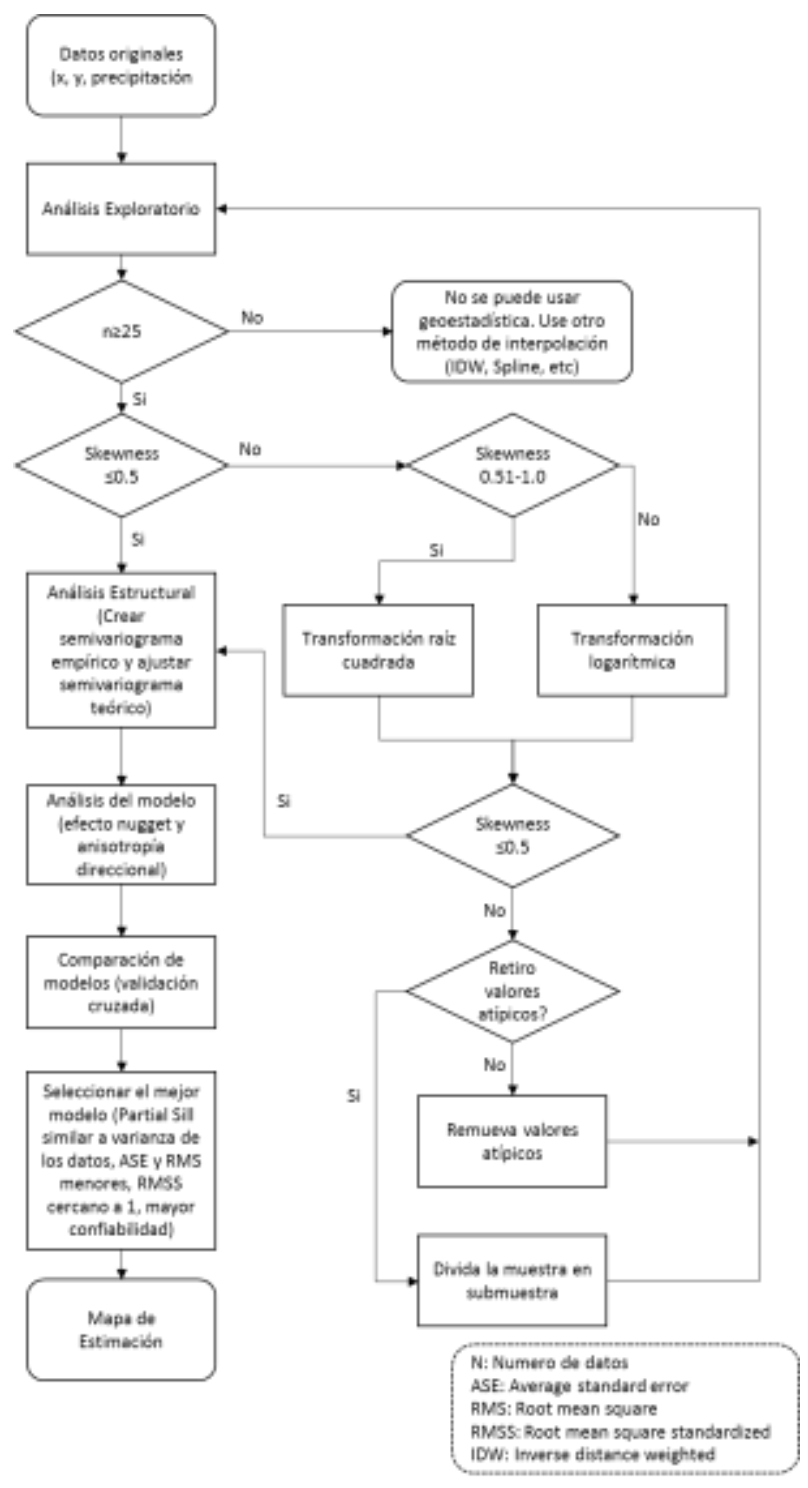

Figura 2. Proceso análisis exploratorio y estructural [20].

\section{Resultados}

En la Tabla 1 se muestran los resultados de la Frecuencia del modelo $\left(F_{A}\right)$ y los años donde se presenta. El modelo con mayor frecuencia es el J-bessel (23), seguido del hole effect (23). Como se observa en la Tabla 1 en el mes de octubre del año 2005 no hay un modelo que se ajuste, debido a que no fue posible encontrar un conjunto de datos con los cuales se lograra la estacionariedad de los mismos, obteniendo valores de desempeño extremadamente bajos.

Para el análisis de los datos de precipitación media mensual se seleccionó un modelo de acuerdo a la Tabla 1 , en el cual se tomó como mejor aquel que se presentara con mayor frecuencia durante los 10 años evaluados. En la Tabla 2, se muestran los errores de estimación promedio para cada mes del año y el modelo seleccionado, es así como para los meses de enero, febrero y julio el mejor es j-bessel con un desempeño de $85.69,87.43$ y 87.88 respectivamente. Para los meses de marzo, abril y junio se escogieron los modelos rational quadratic, tetraspherical y spherical respectivamente. Para los meses de mayo y septiembre se tomó el modelo exponential con un desempeño de 78.08 y 90.57 respectivamente. Para los meses de agosto y octubre el mejor modelo fue el gaussian y el desempeño fue de 80.48 y 91.08 respectivamente. $\mathrm{Y}$, por último, para los meses de noviembre y diciembre el mejor modelo de ajuste fue el hole effect con un desempeño de 75.56 y 81.31 respectivamente. 
Tabla 1. Frecuencias de mejores modelos.

\begin{tabular}{|c|c|c|c|c|c|c|c|c|c|}
\hline \multirow[t]{2}{*}{ Mes } & \multicolumn{9}{|c|}{ Modelos } \\
\hline & $E$ & Ex & $\mathrm{G}$ & $\mathrm{T}$ & $P$ & $\mathrm{H}$ & $\mathrm{R}$ & $\mathrm{J}$ & $\mathrm{K}$ \\
\hline $\mathrm{F}_{\mathrm{A}}$ & 5 & 13 & 21 & 5 & 2 & 23 & 17 & 23 & 9 \\
\hline Ene & & & 2013 & & & $\begin{array}{l}2009 \\
2011\end{array}$ & $\begin{array}{l}2006 \\
2014\end{array}$ & $\begin{array}{l}2007 \\
2008 \\
2010\end{array}$ & $\begin{array}{l}2005 \\
2012\end{array}$ \\
\hline Feb & & 2011 & $\begin{array}{l}2005 \\
2008\end{array}$ & & & 2009 & & $\begin{array}{l}2006 \\
2007 \\
2013 \\
2014\end{array}$ & $\begin{array}{l}2010 \\
2012\end{array}$ \\
\hline Mar & & 2005 & 2013 & & & $\begin{array}{l}2009 \\
2012 \\
2014\end{array}$ & $\begin{array}{l}2006 \\
2008 \\
2011\end{array}$ & $\begin{array}{l}2007 \\
2010\end{array}$ & \\
\hline Abr & & & 2012 & $\begin{array}{l}2007 \\
2009 \\
2013\end{array}$ & 2005 & $\begin{array}{l}2006 \\
2008 \\
2010\end{array}$ & 2011 & 2014 & \\
\hline May & 2012 & $\begin{array}{l}2008 \\
2009 \\
2014\end{array}$ & $\begin{array}{l}2005 \\
2006 \\
2007\end{array}$ & & & 2010 & & 2011 & 2013 \\
\hline Jun & $\begin{array}{l}2011 \\
2014\end{array}$ & $\begin{array}{l}2010 \\
2013\end{array}$ & 2008 & & & $\begin{array}{l}2005 \\
2009\end{array}$ & 2007 & 2006 & \\
\hline Jul & & & $\begin{array}{l}2008 \\
2010\end{array}$ & 2009 & & & $\begin{array}{l}2004 \\
2005 \\
2012\end{array}$ & $\begin{array}{l}2006 \\
2011 \\
2013\end{array}$ & 2007 \\
\hline Ago & & & $\begin{array}{l}2006 \\
2008 \\
2011\end{array}$ & & & $\begin{array}{l}2007 \\
2013\end{array}$ & $\begin{array}{l}2009 \\
2010\end{array}$ & $\begin{array}{l}2004 \\
2005 \\
2012\end{array}$ & \\
\hline Sep & & $\begin{array}{l}2005 \\
2007 \\
2012\end{array}$ & & & & $\begin{array}{l}2010 \\
2013\end{array}$ & $\begin{array}{l}2004 \\
2009\end{array}$ & 2008 & $\begin{array}{l}2006 \\
2011\end{array}$ \\
\hline Oct & 2004 & & $\begin{array}{l}2007 \\
2010 \\
2011 \\
2013\end{array}$ & & 2009 & & & $\begin{array}{l}2006 \\
2008 \\
2012\end{array}$ & \\
\hline Nov & 2006 & $\begin{array}{l}2007 \\
2008\end{array}$ & $\begin{array}{l}2010 \\
2011\end{array}$ & 2013 & & $\begin{array}{l}2004 \\
2005 \\
2009 \\
2012\end{array}$ & & & \\
\hline Dic & & 2006 & 2011 & & & $\begin{array}{l}2007 \\
2009 \\
2012\end{array}$ & $\begin{array}{l}2004 \\
2005 \\
2013\end{array}$ & 2010 & 2008 \\
\hline
\end{tabular}

E: Esférico

Ex: Exponencial

G: Gausiano

T: Traesferico

P: Pentaesferico
H: Holl efecto

R: Racional cuadrático

J: J-Bessel

K: K-Bessel 
Tabla 2. Estadísticos de error de estimación promedio

\begin{tabular}{llllll}
\hline Mes & Modelo & ASE & RMS & RMSS & Desempeño \\
\hline Enero & J-Bessel & 14.81 & 14.31 & 0.99 & 85.69 \\
\hline Febrero & J-Bessel & 14.25 & 12.57 & 1.05 & 87.43 \\
\hline Marzo & Rational & 30.64 & 27.68 & 1.00 & 72.32 \\
& Quadratic & & & & \\
\hline Abril & Tetraspherical & 19.25 & 20.04 & 1.15 & 79.96 \\
\hline Mayo & Exponential & 21.72 & 21.92 & 1.02 & 78.08 \\
\hline Junio & Spherical & 11.97 & 10.51 & 0.98 & 89.49 \\
\hline Julio & J-Bessel & 10.91 & 12.12 & 1.05 & 87.88 \\
\hline Agosto & Gaussian & 18.15 & 19.52 & 1.05 & 80.48 \\
\hline Septiembre & Exponential & 9.33 & 9.43 & 0.99 & 90.57 \\
\hline Octubre & Gaussian & 9.35 & 8.92 & 1.00 & 91.08 \\
\hline Noviembre & Hole Effect & 24.66 & 24.44 & 1.03 & 75.56 \\
\hline Diciembre & Hole Effect & 18.69 & 18.69 & 1.08 & 81.31 \\
\hline
\end{tabular}

Usando pruebas de análisis de varianza (ANOVA), se estudiaron los parámetros de los modelos seleccionados que fueron agrupados mes a mes. Para esto se tomaron como variables del modelo los parámetros nugget, major range y partial sill. Estas variables se caracterizan por ser independientes, cuantitativas y en razón a la escala.

Inicialmente se analiza si la distribución de las variables en los meses, es normal o no. Para esto se evalúan tres parámetros:

- Similitud entre las medidas de tendencia central del conjunto de datos analizados

- El coeficiente de sesgo debe ser $\leq 0.5$

- La desviación estándar debe estar entre 0 y 1

En la Tabla 3 se puede observar la autocorrelación espacial de los puntos de muestra medidos extraídos del semivariograma, el cual consistió en un trazado de cada par de ubicaciones (estaciones meteorológicas), que se ajustaron al mejor modelo. En este se pudo observar que en una determinada distancia el modelo se nivelaba. En la distancia donde el modelo comenzaba a nivelarse aparecía el major range y en el valor en el cual el modelo de semivariograma alcanza el major range (valor de eje) se denomina sill. $Y$ un partial sill es una sill menos un nugget. Este nugget es denominado efecto nugget y se atribuye a errores de medición o a fuentes espaciales de variación a distancias que son menores que el intervalo de muestreo (o ambas cosas), en los fenómenos naturales pueden variar espacialmente en un rango de escalas.
Tabla 3. Análisis de la autocorrección espacial de los puntos medidos.

\begin{tabular}{lllll}
\hline Mes & Modelo & Nugget & $\begin{array}{l}\text { Major } \\
\text { Range }\end{array}$ & $\begin{array}{l}\text { Partial } \\
\text { sill (Gs) }\end{array}$ \\
\hline Enero & J-Bessel & 298.79 & 113527.50 & 427.62 \\
\hline Febrero & J-Bessel & 2.73 & 92598.08 & 5.73 \\
\hline Marzo & $\begin{array}{l}\text { Rational } \\
\text { Quadratic }\end{array}$ & 48.03 & 71013.24 & 1412.59 \\
\hline Abril & Tetraspherical & 1247.43 & 87194.11 & 412.70 \\
\hline Mayo & Exponential & 599.40 & 98184.22 & 2956.73 \\
\hline Junio & Spherical & 2.75 & 65593.07 & 3.21 \\
\hline Julio & J-Bess & 79.05 & 103600.19 & 152.72 \\
\hline Agosto & Gaussian & 373.69 & 79195.73 & 880.36 \\
\hline Septiembre & Exponential & 99.23 & 89014.92 & 949.08 \\
\hline Octubre & Gaussian & 366.21 & 95604.55 & 215.02 \\
\hline Noviembre & Hole Effect & 1419.72 & 106389.19 & 2744.96 \\
\hline Diciembre & Hole Effect & 681.68 & 95167.49 & 1918.66 \\
\hline
\end{tabular}

En la Tabla 4 se muestra un análisis descriptivo de los grupos, que permiten reconocer que tanto se dispersan los datos, indicando cuanto se desvían las observaciones alrededor de la media. Para el caso de los datos analizados, se evidencia el déficit de precipitación para las diferentes escalas temporales que se presentan en la zona de estudio y de esta manera poder evaluar el déficit de precipitación sobre la disponibilidad de los distintos tipos de recursos hídricos.

Tabla 4. Análisis descriptivo de los meses evaluados

\begin{tabular}{llll}
\hline Mes & Modelo & Media & $\begin{array}{l}\text { Desviación } \\
\text { estándar }\end{array}$ \\
\hline Enero & J-Bess & 71.67 & 35.17 \\
\hline Febrero & J-Bess & 94.02 & 45.78 \\
\hline Marzo & Rational & 169.37 & 63.69 \\
& Quadratic & & \\
\hline Abril & Tetraspherical & 241.99 & 66.84 \\
\hline Mayo & Exponential & 282.88 & 71.44 \\
\hline Junio & Spherical & 198.95 & 46.70 \\
\hline Julio & J-Bess & 181.66 & 48.28 \\
\hline Agosto & Gaussian & 204.56 & 54.35 \\
\hline Septiembre & Exponential & 212.21 & 49.78 \\
\hline Octubre & Gaussian & 269.89 & 73.71 \\
\hline Noviembre & Hole Effect & 249.09 & 82.37 \\
\hline Diciembre & Hole Effect & 145.20 & 59.13 \\
\hline
\end{tabular}

El análisis espacio temporal de la precipitación realizado a partir de los datos pluviométricos de estaciones hidrometeorológicas existentes en el área de estudio del Cañón del Rio Cauca, permitió caracterizar la variabilidad de las lluvias y la distribución de las mismas en el periodo comprendido 2004 - 2014 (Fig. 3), encontrando un comportamiento bimodal de periodos secos y lluviosos (estacionalidad), primero un periodo seco comprendido entre los meses de diciembre a 
febrero y un segundo periodo entre junio a agosto, así mismo dos periodos lluviosos comprendidos entre los meses de marzo a mayo y septiembre a noviembre. Lo anterior muestra una correspondencia de la distribución temporal de la lluvia, comparado con el comportamiento de la precipitación en Colombia establecido por el IDEAM.

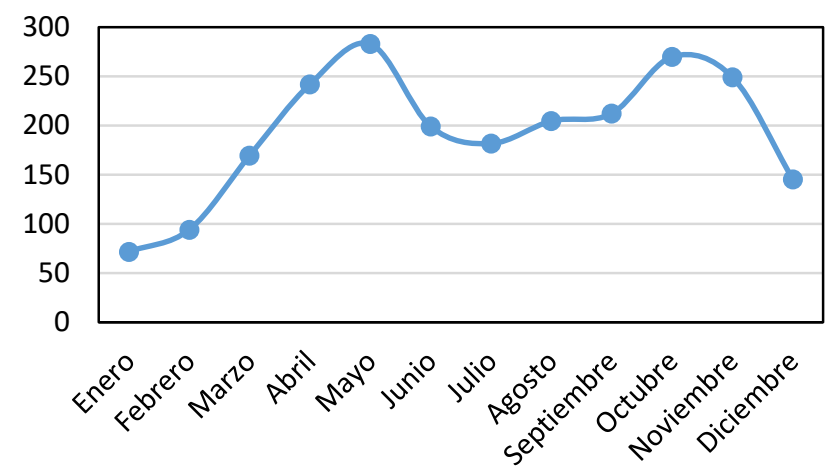

Figura 3. Precipitación promedio mensual ( $\mathrm{mm} / \mathrm{mes})$ en el área de estudio en el periodo comprendido entre 2004-2014.

Con relación a la distribución espacial de la precipitación en el área de estudio del Cañón del Rio Cauca, se identifica un patrón de distribución en todos los meses que evidencia una gradación de las precipitaciones de menor a mayor en sentido norte a sur (Figura 4 a la Figura 15). La zona norte del cañón del Rio Cauca se encuentra asociada a la subregión del occidente antiqueño, comprendida por los municipios de Anzá, Ebéjico, Caicedo, Santa Fe de Antioquia, Sopetrán, San Jerónimo, Olaya, Liborina, Buriticá y Sabanalarga, mientras que la zona sur se encuentran los municipios que conforman gran parte del suroeste antioqueño, destacándose los municipios de Venecia, Fredonia, Tarso, Jericó, Andes, Jardín, Támesis, Valparaíso.

En la Figura 4 a la Figura 15 se puede identificar como la zona de estudio es dividida claramente por una zona de transición, la cual se encuentra comprendida entre los municipios de Betulia, Armenia, Heliconia, Medellín y Bello, que hace que coincidan cambios de temperatura y coberturas vegetales existentes entre ambas subregiones.

En el estudio se evaluaron nueve modelos teóricos de semivariogramas para cada mes de precipitación media, de los cuales los más importantes fueron j-bessel, hole effect y gaussian, que no coinciden con los resultados de la literatura que reportan los modelos esféricos como los mejores. Estos resultados en parte si coinciden con el estudio realizado por [19], que encontraron el j-bessel $y$ el hole effect como los modelos teóricos de mejor ajuste.

Como comentan Londoño et al, la ventaja de un modelo geoestadístico radica en la información adicional que este aporta sobre el máximo cambio de la varianza de los datos con la distancia (partial sill) y el alcance de la autocorrelación espacial de los mismos (major range). Analizando la frecuencia de los modelos, se observa que los modelos j-bessel, hole effect y gaussian representan el $57 \%$ de los meses estudiados siendo ligeramente más frecuente j-bessel y hole effect en 23 meses con respecto al gaussian en 21 meses.

Para el evaluar el comportamiento en todos los meses no se pudo tomar solo estos tres modelos de mayor frecuencia, sino que se tuvo que evaluar mes a mes, por esto para los meses de enero, febrero y julio se encontró que los criterios de validación cruzada eran buenos para el modelo j-bessel por lo que se optó por representar la variable precipitación en dichos meses de estudio, obteniendo en general un desempeño de estimación promedio del $68 \%$ como se observa en las Figura 4 , Figura 5 y Figura 9.

Para el mes de marzo se tomó como mejor modelo el rational quadratic según los criterios de validación cruzada, los cuales eran buenos con un desempeño de estimación de $52 \%$. Para los meses de abril y junio se tomaron los modelos tetraspherical (51\%) y spherical (61\%). Para los meses de mayo y septiembre se tomó como mejor modelo el exponential con un desempeño de estimación promedio de $56 \%$. Para los meses de agosto y octubre se tomó como mejor modelo el gaussian y para los meses de noviembre y diciembre el hole effect, estos dos últimos modelos con desempeño de estimación promedio de 51 y $52 \%$ respectivamente. Su representación de evidencia en las Figura 6 a Figura 15.

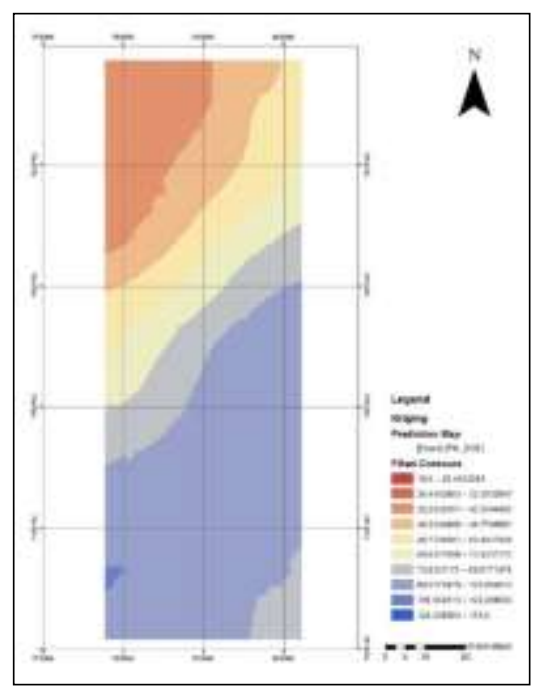

Figura 4. Caracterización espacial de la variable precipitación para el mes de enero 


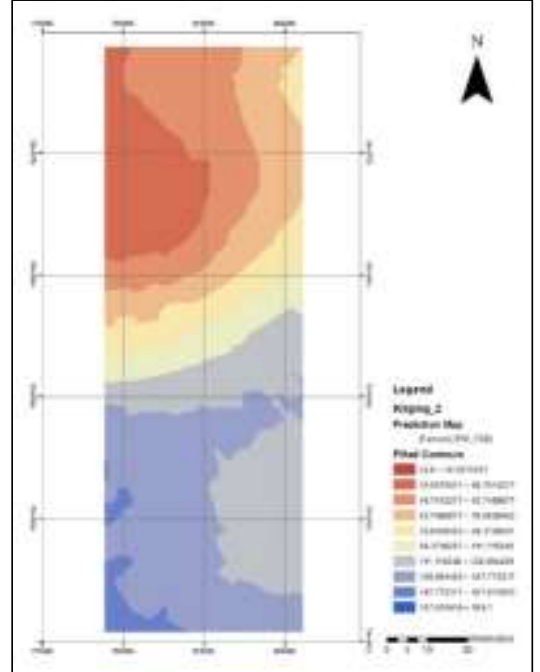

Figura 5. Caracterización espacial de la variable precipitación para el mes de febrero

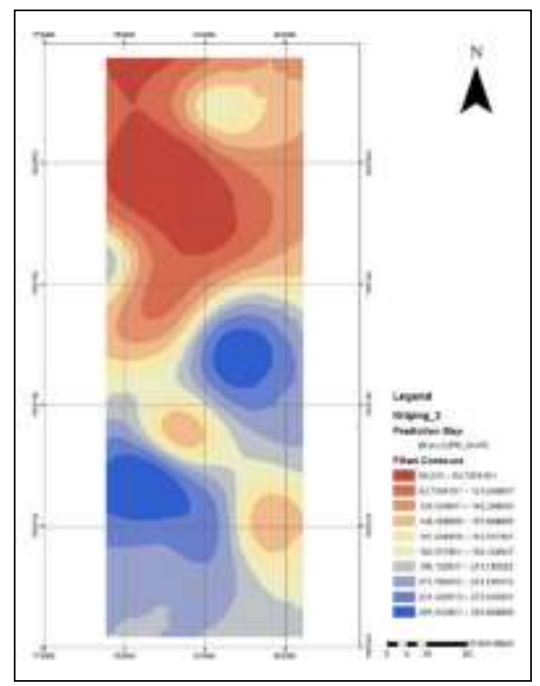

Figura 6. Caracterización espacial de la variable precipitación para el mes de marzo

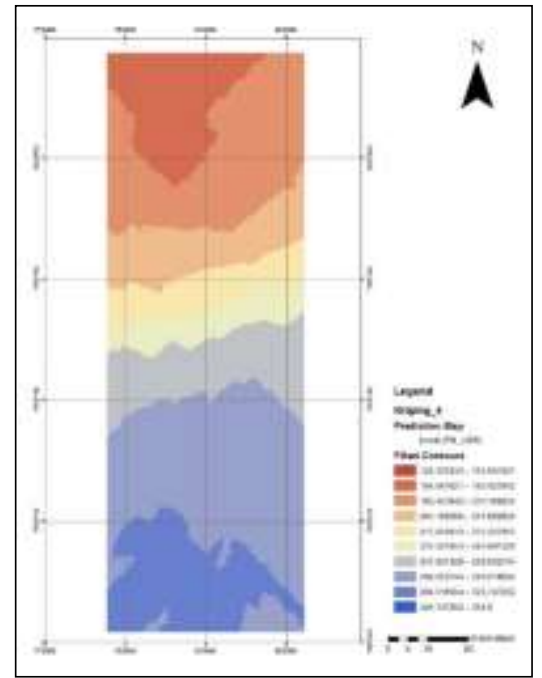

Figura 7. Caracterización espacial de la variable precipitación para el mes de abril

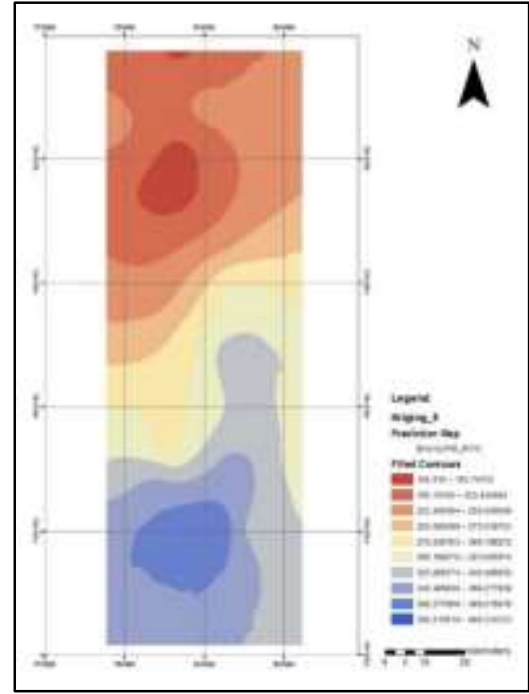

Figura 8. Caracterización espacial de la variable precipitación para el mes de mayo

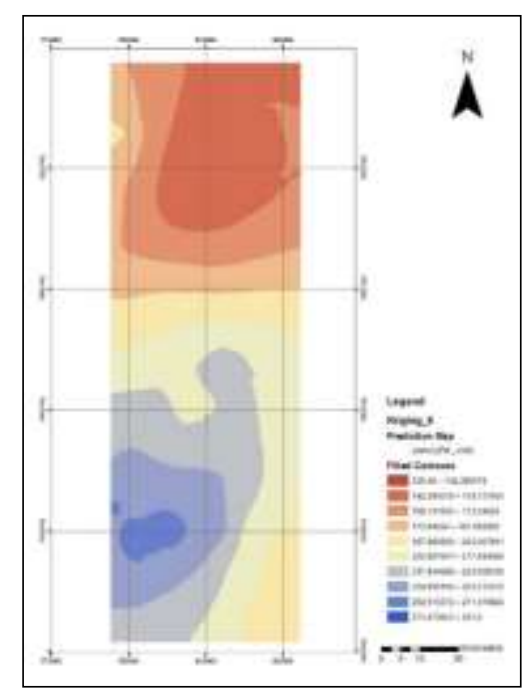

Figura 9. Caracterización espacial de la variable precipitación para el mes de junio

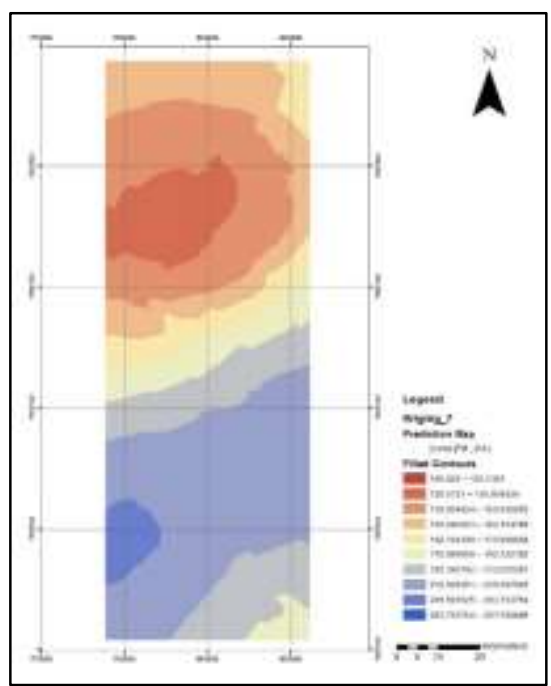

Figura 10. Caracterización espacial de la variable precipitación para el mes de julio 


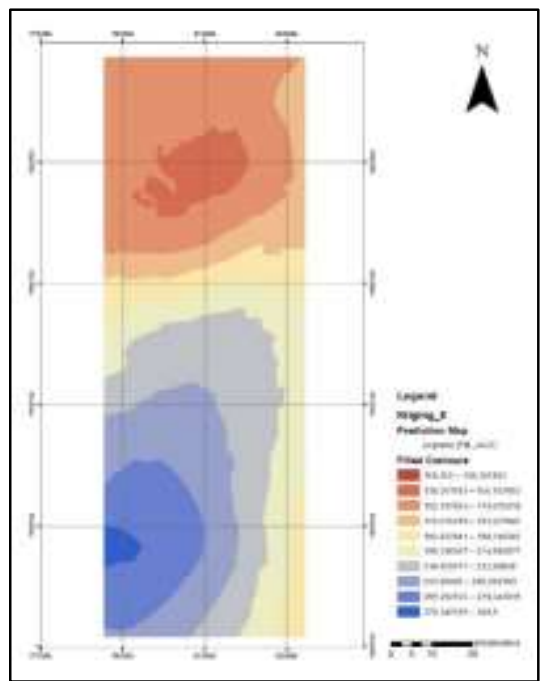

Figura 11. Caracterización espacial de la variable precipitación para el mes de agosto

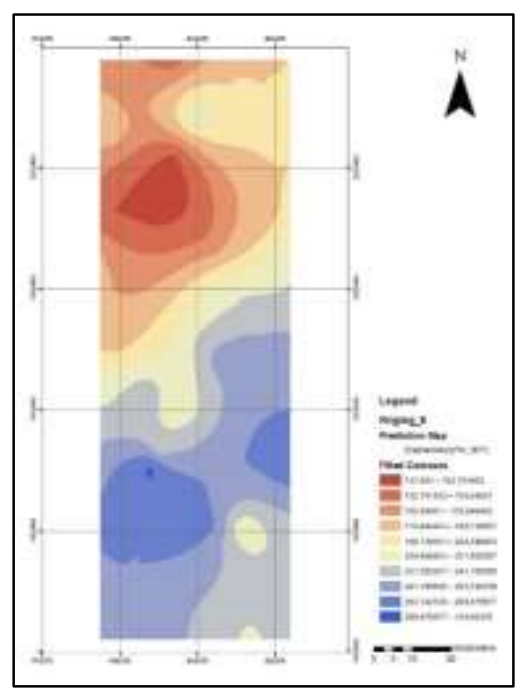

Figura 12. Caracterización espacial de la variable precipitación para el mes de septiembre

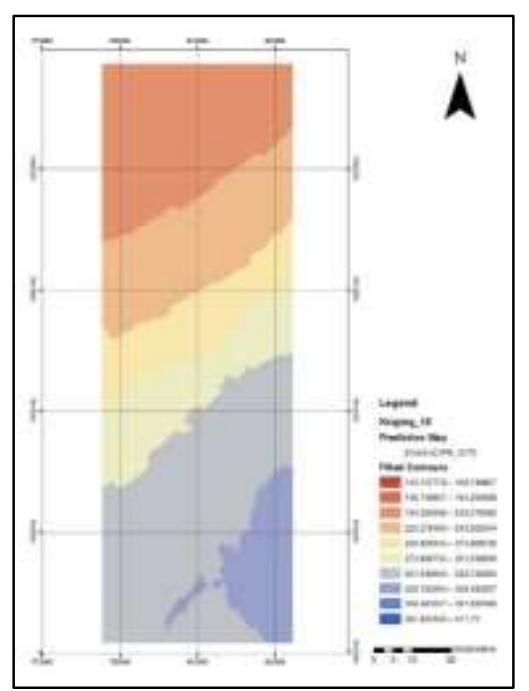

Figura 13. Caracterización espacial de la variable precipitación para el mes de octubre

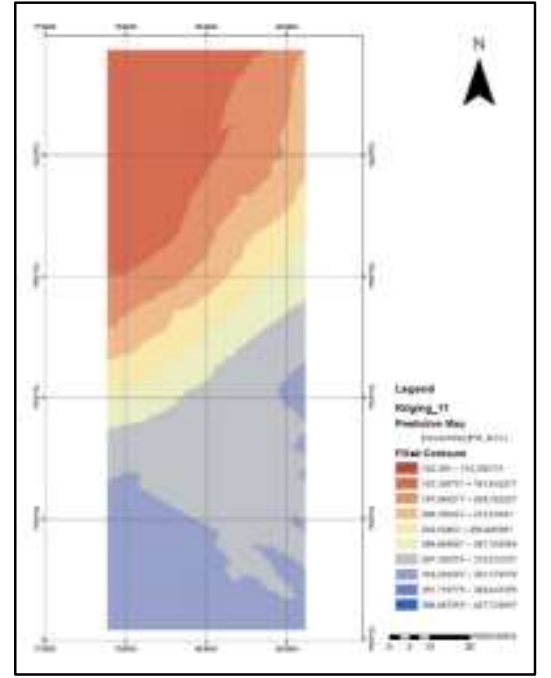

Figura 14. Caracterización espacial de la variable precipitación para el mes de noviembre

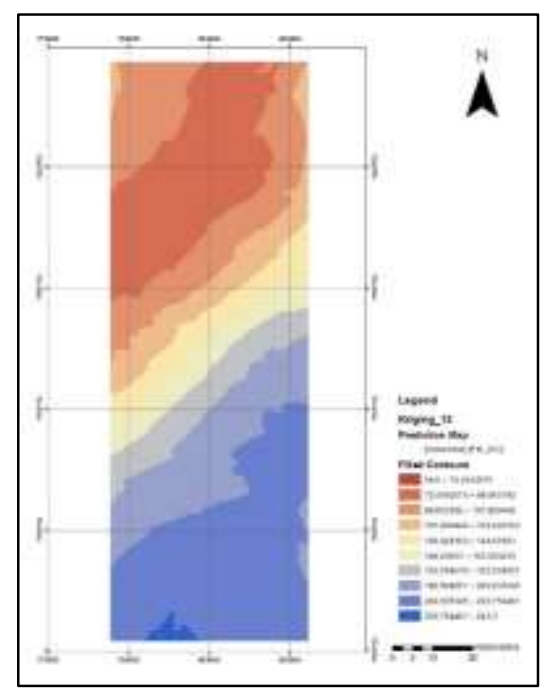

Figura 15. Caracterización espacial de la variable precipitación para el mes de diciembre

Se puede observar que en casi todos los modelos aplicados se tiene un desempeño por debajo de $80 \%$, esto se debe a que en muchos de ellos la desviación estándar es alta, lo cual afecta el desempeño del modelo. Este efecto se produce por la distribución ligeramente asimétrica positiva, la cual se debe a la presencia de valores extremos superiores a la media, reflejando la naturaleza de la variable. Otro factor que posiblemente puede influir es la baja presencia de estaciones en el área del estudio, que implica una menor cantidad de datos para alimentar los modelos.

En cuanto a la caracterización espacial de la variable precipitación se muestra una similitud entre todos los meses, observándose así, como de norte a sur la precipitación va aumentando, evidenciándose dos zonas claramente definidas de precipitación, que se puede deber a la cobertura vegetal, la temperatura y pequeñas zonas de microcuencas topográficas que pueden estar favoreciendo corrientes de vientos que llevan a que las precipitaciones aumenten. 


\section{Conclusiones}

Los mapas de precipitación obtenidos fueron el resultado de una combinación óptima entre los estimados a partir de las bases de datos del IDEAM de precipitación media mensual y los estimados a partir de la implementación de los modelos aplicados para cada mes. Dicha combinación o ponderación se basó en los campos de varianza del error.

Se encontró que los modelos j-bessel, hole effect y gaussian fueron los que presentaron mayor frecuencia de los modelos evaluados, siendo el j-bessel y el hole effect los de mayor frecuencia con 23 meses cada uno. A pesar de estos efectos ambos solo fueron aplicados en los meses de enero, febrero y julio para el caso del jbessel y los meses de noviembre y diciembre para el hole effect.

La metodología llevada a cabo permitió reconstruir el comportamiento espacial de la variable precipitación obteniendo resultados coherentes y considerando la topografía como un factor fundamental para explicar la variabilidad en la zona de estudio (Cañón del Rio Cauca).

Por último, se pudo evidenciar que la distribución espacial de la precipitación en el área de estudio del Cañón del Rio Cauca, se identifica un patrón de distribución en todos los meses que evidencia una gradación de la precipitaciones de menor a mayor en sentido norte a sur, dividiendo el área de estudio en dos zonas, una fuertemente marcada por menores precipitaciones en el norte, asociada a la subregión del occidente antioqueño y otra marcada por el aumento de las precipitaciones promedio mensuales, asociado a la subregión del suroeste antioqueño.

Para lograr un mayor entendimiento la variable precipitación y de otras variables climáticas es necesario realizar análisis más profundo que lleve a comprender mejor el clima colombiano, el método desarrollo puede apoyar este tipo de análisis.

A pesar de que en Colombia la precipitación es una de las variables mejor observada, la red que permite capturar información deberá en el futuro mejorarse, toda vez que su densidad, distribución y operación presente algunas deficiencias, más aún si se consideran los grandes gradientes altitudinales y los cambios inducidos por la topografía y la climatología.

Los métodos de interpolación pueden generar extrapolaciones no deseadas, pues la variable en la realidad puede tener un comportamiento algo diferente. En particular la precipitación se caracterizada por una gran variabilidad espacio temporal, incluidas escalas de tiempo mensual o estacional, interpolar usando métodos como Kriging no garantiza resultados que permitan describir lo que sucede con la variable en cada instante y en todo lugar.

\section{Trabajos futuros}

Continuar con trabajos que realicen comparaciones entre las mediciones directas e indirectas de variables hidrológicas constituyen áreas de investigación que a futuro deben continuar desarrollándose a fin de poder lograr la mejor representatividad en el espacio y el tiempo dichas variables y con ello, lograr un adecuado modelamiento de las mismas.

\section{Referencias}

[1] Y. Cai, W. Yue, L. Xu, Z. Yang, and Q. Rong, "Sustainable urban water resources management considering life-cycle environmental impacts of water utilization under uncertainty," Resour. Conserv. Recycl., vol. 108, pp. 21-40, 2016.

[2] Y. Chen, S. Zhang, Y. Zhang, L. Xu, Z. Qu, G. Song, and J. Zhang, "Comprehensive assessment and hierarchical management of the sustainable utilization of urban water resources based on catastrophe theory," J. Taiwan Inst. Chem. Eng., vol. 60, pp. 430-437, 2016.

[3] D. Hering, L. Carvalho, C. Argillier, M. Beklioglu, A. Borja, A C. Cardoso, H. Duel, T. Ferreira, L. Globevnik, J. Hanganu, S. Hellsten, E. Jeppesen, V. Kode??, A. L. Solheim, T. N??ges, S. Ormerod, Y. Panagopoulos, S. Schmutz, M. Venohr, and S. Birk, "Managing aquatic ecosystems and water resources under multiple stress - An introduction to the MARS project," Sci. Total Environ., vol. 503-504, pp. 10-21, 2015.

[4] H. Lévite, H. Sally, and J. Cour, "Testing water demand management scenarios in a water-stressed basin in South Africa: Application of the WEAP model," Phys. Chem. Earth, vol. 28, no. 20-27, pp. 779-786, 2003.

[5] C. Kidd, V. Levizzani, J. Turk, and R. Ferraro, "Satellite precipitation measurements for water resource monitoring," $\mathrm{J}$ Am. Water Resour. Assoc., vol. 45, no. 3, pp. 567-579, 2009.

[6] A. Y. Hou, R. K. Kakar, S. Neeck, A. A. Azarbarzin, C. D. Kummerow, M. Kojima, R. Oki, K. Nakamura, and T. Iguchi, "The global precipitation measurement mission," Bull. Am. Meteorol. Soc., vol. 95, no. 5, pp. 701-722, 2014.

[7] V. M. Mantas, Z. Liu, C. Caro, and A. J. S. C. Pereira, "Validation of TRMM multi-satellite precipitation analysis (TMPA) products in the Peruvian Andes," Atmos. Res., vol. 163, pp. 132-145, 2015.

[8] F. J. Tapiador, F. J. Turk, W. Petersen, A. Y. Hou, E. GarcíaOrtega, L. A. T. Machado, C. F. Angelis, P. Salio, C. Kidd, G. J. Huffman, and M. de Castro, "Global precipitation measurement: Methods, datasets and applications," Atmos. Res., vol. 104-105, pp. 70-97, Feb. 2012.

[9] P. Salio, M. P. Hobouchian, Y. García Skabar, and D. Vila, "Evaluation of high-resolution satellite precipitation estimates over southern South America using a dense rain gauge network," Atmos. Res., vol. 163, pp. 146-161, 2015.

[10] M. y E. A. Instituto de Hidrología, Estudio Nacional del Agua 2014. 2014

[11] D. dos R. Pereira, M. A. Martinez, D. D. da Silva, and F. F. Pruski, "Hydrological simulation in a basin of typical tropical climate and soil using the SWAT Model Part II: Simulation of hydrological variables and soil use scenarios," J. Hydrol. Reg. Stud., vol. 5, pp. 149-163, 2016.

[12] O. Mejía Rivera, J. Correa Giraldo, F. González Maya, and D. Montoya Velilla, "Un Modelo para la Administración de la Demanda del Recurso Hídrico Superficial en la Jurisdicción de Corantioquia (SGDH - Duberdicus)," p. 76, 2006.

[13] Corporación Autónoma del Centro de Antioquia, Territorio Corantioquia. 2011.

[14] M. y E. A. Instituto de Hidrología, "Lineamientos conceptuales 
y metodológicos para la evaluación regional del agua-ERA 2013," 2013.

[15] O. J. Mesa Sánchez, G. Poveda Jaramillo, J. I. Vélez Upegui, J. F. Mejía Valencia, C. D. Hoyos Ortiz, R. Mantilla Gutiérrez, O. J. Barco Mejía, L. A. Cuartas Pineda, B. Botero Hernández, and M. I. Montoya, "Distribución espacial y ciclos anual y semianual de la precipitación en Colombia.," XIV Semin. Hidráulica e Hidrol., no. 1, pp. 1-9, 2000.

[16] J. U. Arango Arroyave, "Etnobotánica Asociada Al Barequeo En El Cañón Medio Del Río Cauca: Jurisdicción De Los Municipios De Peque Y Sabanalarga. Occidente Antioqueño," Boletín Ciencias la Tierra, no. 35, pp. 45-51, 2014.

[17] L. R. Holdridge, Ecología basada en zonas de vida. Instituto Interamericano de Ciencias Agrícolas, 1982.

[18] L. S. Espinal, "Geografia ecologica del departamento de Antioquia (Zonas de vida (formaciones Vegetales) del departamento De Antioquia)," Fac. Nac. Agron., vol. 24, no. 60, pp. 24-32, 1964.

[19] L. A. Londoño, J. E. Cañon, R. D. Villada, and Li. Y. López, "Caracterización espacial de PM10 en la ciudad de Medellín mediante modelos geoestadísticos Spatial characterization of pm10 in Medellín Colombia by geostatistical models," Ing.
Uniersidad San Buena Ventur. sede Medellin, vol. 6, no. 2, pp. 26-35, 2015.

[20] O. M. Quiroz Londono, D. E. Martinez, H. E. Massone, L. A. Londono Ciro, and C. Dapena, "Spatial distribution of electrical conductivity and stable isotopes in groundwater in large catchments: a geostatistical approach in the Quequen Grande River catchment, Argentina.," Isotopes Environ. Health Stud., vol. 51, no. 3, pp. 411-425, 2015.

[21] R. W. M. A. Oliver, Geostatistics for Environmental Scientists. 2007.

[22] A. Pollice and G. J. Lasinio, "Web Working Papers by The Italian Group of Environmental Statistics Two approaches to imputation and adjustment of air quality data from a composite monitoring network Two approaches to imputation and adjustment of air quality data from a composite monito," Statistica, no. March, 2008.

[23] I. C. Villada Arias and L. A. Londoño Ciro, "Aplicación de métodos geoestadísticos para la caracterización de la calidad química de un depósito de material calcáreo," Boletín Ciencias la Tierra, vol. 1, no. 35, pp. 15-24, 2014. 